\title{
Blockchain y arbitraje: un nuevo enfoque en la resolución de disputas. Especial énfasis en smartcontracts y criptodivisas
}

\author{
Blockchain and Arbitration: A New Approach to Dispute Resolution. \\ Special Emphasis on Smartcontracts and Cryptocurrencies
}

\author{
Ignacio Tasende \\ ORCID: 0000-0002-3728-6394 \\ Universidad de la República, Uruguay \\ Correo: i.tasende@hotmail.com
}

Recibido: $12 / 04 / 2020$

Aceptado: 03/11/2020

Resumen: Las nuevas tecnologías han revolucionado el mundo de los negocios y servicios. Sin embargo, las transacciones efectuadas mediante o con el auxilio de estas tecnologías no están exentas de problemas. Este artículo aborda el funcionamiento de la blockchain, una de las tecnologías que, pese a tener un futuro incierto, desde hace tiempo se viene aplicando. La blockchain, según estimaciones, transformará la forma en que se realizan transacciones e incidirá, notoriamente, tanto en la modalidad de prestación de servicios profesionales como en la resolución de controversias. En este trabajo se trata su vínculo con el arbitraje en cuanto método de resolución de controversias más adecuado a tales efectos, con especial énfasis en aquellos aspectos prácticos que podrían dar lugar a conflictos para cuya solución no hay un marco regulatorio establecido.

Palabras clave: arbitraje; tecnologías; blockchain; smartcontracts; criptomonedas.

\begin{abstract}
New technologies have revolutionized the world of business and services. Nonetheless, transactions carried out using or with the help of these technologies are not exempt of problems. This article addresses the way in which blockchain works, one of the technologies that, despite having an uncertain future, is already being applied. Blockchain is expected to revolutionize the way in which transactions are conducted and will have a significant impact on both the way professional services are provided and on the resolution of disputes. This work also analizes its link with arbitration, as the most appropriate method of dispute resolution for such purposes, with emphasis on practical aspects that could give rise to conflicts for which there is no regulatory framework.
\end{abstract}

Keywords: arbitration; technologies; blockchain; smartcontracts; cryptocurrencies. 


\section{Prefacio}

Sin perjuicio del entorno aparentemente infalible y sin riesgos que representa la tecnología blockchain, sus múltiples beneficios no obstan a la aparición de controversias, pues no es posible que el comportamiento humano sortee todo riesgo de disputa (Katsh y Rabinovich-Einy, 2017). En este artículo se abordarán las complejidades que se pueden plantear a nivel regulatorio.

\section{Blockchain: el auge de la tecnología en bloques}

\section{Aproximaciones}

La blockchain, o cadena de bloques, se ha promocionado como la nueva tecnología infalible que puede utilizarse para absolutamente todo, desde criptodivisas, hasta registros catastrales, documentos de identidad y registros sanitarios (Hodge y Neally, 2018). Sus promotores estiman que generará un cambio sustancial, garantizando a través de la eliminación de los intermediarios tradicionales, como por ejemplo, los bancos, tanto la seguridad de los datos, como su autenticidad (De Filippi y Wright, 2015). Esta "nueva internet" (Fenwick et al., 2017), se afirma, cambiará la manera en que se realizan las transacciones, fortaleciendo los compromisos y asegurando una ejecución sin problemas (Bacon et al., 2017).

La cadena de bloques es un registro único, consensuado y distribuido en varios "nodos" de una red. A modo de analogía, la blockchain se parece a un gran libro contable, donde se registra cada transacción. Cada bloque almacena una cantidad de registros o transacciones, información relativa al mismo y su relación tanto con el bloque anterior de la cadena como con el siguiente. Esto, a través del hash, es decir, un código que constituye una suerte de huella digital. Cada bloque tiene una posición concreta en la cadena, la que es inamovible, puesto que esos bloques contienen información del hash del bloque anterior.

La cadena completa se guarda en cada "nodo" o computadora de la red que conforma la blockchain, es decir, se almacena una copia exacta de la cadena en todos los participantes de la red. A medida que se crean nuevos registros, estos son, primeramente, verificados y validados por los nodos de la red y luego añadidos a un nuevo bloque que se enlaza a la cadena (Pastorino, 2018). 
En definitiva, se trata de un libro descentralizado de transacciones, mantenido por usuarios y no por un tercero de confianza, que funciona principalmente para el intercambio de activos digitales. Las transacciones en la cadena se dividen en "bloques" cifrados, irreversibles, compartidos y verificados por los usuarios. Cada uno de esos bloques, cuya modificación es imposible, se enlaza o encadena al siguiente, mediante firmas criptográficas, asegurándose su validez y evitando la manipulación.

Hasta la fecha, y en una primera etapa, la moneda virtual bitcoin representa el caso más exitoso de uso de la tecnología blockchain, dando lugar al auge de las criptomonedas (Bartoletti y Pompianu, 2017). En una segunda etapa, nacen los smartcontracts o contratos inteligentes. Simultáneamente, se comienza a observar un creciente interés de los Estados por el uso de estas tecnologías. En ese sentido, Estonia y Suecia, por ejemplo, encabezaron los esfuerzos por implementar la tecnología blockchain en la esfera gubernamental, experimentando con registros de datos basados en la cadena de bloques, entre otros.

La tecnología blockchain puede incluso ser útil en materia de ciberseguridad, mejorando potencialmente la seguridad cibernética al impedir tareas fraudulentas y al detectar la manipulación de información.

\section{Principales características}

Si bien existen cadenas de bloque centralizadas, donde una autoridad central elige y distribuye el siguiente bloque de la cadena, y cadenas que no son totalmente anónimas, a continuación, se analizarán someramente los principales rasgos de la blockchain: descentralización, inmutabilidad y anonimato (Demartini et al., 2018; Gupta, 2018).

\section{Descentralización}

La idea central de esta tecnología es eliminar el rol del tercero intermediario, lo cual permite que las transacciones se realicen y documenten de forma descentralizada y distribuida. Esto se logra distribuyendo las funciones que en el pasado ocupaba un solo organismo a múltiples actores, permitiendo que los usuarios realicen cada una de esas funciones, siempre que sus conocimientos tecnológicos e infraestructura se lo permitan.

En una transacción monetaria tradicional, el banco verifica la identidad de las partes, la disponibilidad de los montos cuya transferencia se asumió y supervisa la documentación. Por el contrario, en una operación económica con (por ejemplo) bitcoins, 
los esfuerzos conjuntos de "nodos" y "mineros" son los que garantizan que la persona que transfiere los fondos realmente los tiene y que el destinatario es el correcto (Weber, 2018; Pilkington, 2016). Lo mismo ocurre con el registro y documentación de la transacción que, en todas las computadoras, será idéntico.

Los "nodos" son computadoras. Los usuarios verifican la validez de cada transacción y si la mayoría de ellos dice que la transacción es válida, queda entonces escrita en un bloque. Si una transacción es alterada, las otras computadoras tendrán el "hash" original y no permitirán que esa alteración o manipulación ocurra.

Por su parte, los “mineros", es decir las personas que crean nuevos bloques en la cadena y verifican los bloques que fueron añadidos a cambio de una remuneración, garantizan la exactitud de las transacciones.

\section{Inmutabilidad}

Una vez que los bloques de la cadena son aprobados se vuelven inmutables e irreversibles. No hay posibilidad de alterar una transacción registrada en la cadena. Tratándose de contratos inteligentes, la inmutabilidad se vincula además con la ejecución del contrato. En efecto, los smartcontracts se documentan en códigos informáticos que dan lugar a una ejecución automática una vez que se cumple una condición preestablecida, que permite arribar a un resultado inequívoco.

\section{Anonimato}

A través de esta tecnología se posibilita el intercambio anónimo, que garantiza la seguridad e integridad de los datos mediante el método del encriptado. El anonimato, la inmutabilidad y la descentralización son elementos que, combinados, contribuyen en la creación del equilibrio que habilita la eliminación de los intermediarios. La confianza en las entidades e individuos se sustituye por procesos fijos, criptografía compleja y documentación inmutable.

\section{Resolución de disputas online y blockchain}

La naturaleza consensual de los mecanismos alternativos de resolución de disputas (ADR: alternative dispute resolution) como el arbitraje, permite a las partes un amplio margen de flexibilidad en la ordenación del proceso, lo que también aplica al uso de herramientas informáticas. En ese sentido, los instrumentos internacionales sobre 
arbitraje establecen que las partes pueden acordar el procedimiento que el Tribunal deberá seguir y que, a falta de tal acuerdo, el tribunal dirigirá el procedimiento como lo crea apropiado (Ley Modelo UNCITRAL, 1985; Reglamento ICC, 2017), fomentando la utilización de tecnologías (Reglamento ICDR, 2014).

Los procedimientos de resolución de conflictos en línea (ODR: online dispute resolution), por su parte, surgieron hace décadas junto con el creciente desarrollo del comercio electrónico o e-commerce. Naturalmente, los tribunales tradicionales no eran viables a efectos de resolver controversias que, en su mayoría, versaban sobre montos de dinero ínfimos, entre personas de distintos países. Como respuesta a esa dificultad es que surgen precisamente los ODR. En ese orden, en los últimos años se han impulsado proyectos de ODR basados en tecnología blockchain. Sin perjuicio de que su análisis excede el propósito de este artículo, se mencionará sucintamente la mecánica de una de las plataformas que incorpora estos servicios de resolución de disputas en línea, bajo la tecnología de la cadena de bloques.

Una de las plataformas más desarrolladas en este sentido, es Kleros. Kleros es una aplicación descentralizada construida sobre Ethereum, que funciona como un tercero descentralizado para arbitrar disputas en todo tipo de contratos. Se basa en los incentivos de la teoría del juego para que los jurados decidan los casos correctamente. El resultado es un sistema de resolución de disputas que emite juicios finales de forma rápida, barata, fiable y descentralizada (Ast, George y Lesaege, 2019).

En caso de que se suscite una controversia relativa a la ejecución del contrato, se congelan las transferencias de fondos bajo este, hasta que la disputa sea resuelta. Ello sólo será posible en la medida en que las partes hayan preseleccionado en el contrato a Kleros, como plataforma para la resolución de conflictos y, asimismo, hayan establecido determinadas cuestiones relativas al procedimiento como, por ejemplo, el número de integrantes de un "jurado" (Ast, 2017).

Si una de las partes entiende que el contrato fue incumplido, debe enviarle las pruebas correspondientes a Kleros, aseguradas mediante un encriptado. La resolución se logra mediante la votación del jurado, cuya remuneración debe ser depositada para que inicie el proceso. Los jurados son anónimos y el proceso de selección es al azar, mediante el uso de "fichas" (tokens: unidades de valor). A medida que se inscriben los jurados, son asignados a tribunales específicos en virtud de sus cualidades. Estos reciben las pruebas, 
las evalúan, ponderan y resuelven. Naturalmente, el fallo debe ser fundado, y se ejecuta automáticamente.

Este procedimiento, útil en determinadas circunstancias como, por ejemplo, en aquellos casos en que consumidores disputan montos ínfimos desde grandes distancias, presenta, sin embargo, algunas dificultades desde una perspectiva constitucional, a la luz de los principios del debido proceso, en la medida en que en la automatización de procesos no están presentes elementos de índole subjetiva cuya ausencia puede derivar en soluciones injustas. Sin embargo, la mayoría de posibles violaciones al debido proceso son sorteadas en estos procedimientos, por ejemplo, mediante notificaciones adecuadas, intercambio de comentarios de las partes automáticamente ejecutados, correcta composición del tribunal, cuya imparcialidad e independencia está garantizada, etc.

En definitiva, la innegable utilidad de los procedimientos de resolución de disputas en línea parece presumir que estos mecanismos seguirán desarrollándose cada vez más, y los abogados deberán adaptarse a esa realidad.

\section{Smartcontracts: del papel a la computadora}

\section{¿Qué son?}

Los contratos inteligentes utilizan la tecnología blockchain y son acuerdos que se caracterizan por la ejecución automática de instrucciones electrónicas redactadas en un código informático. Así, los smartcontracts funcionan bajo la lógica de "if - then", permitiendo que se autoejecuten las transferencias de fondos una vez que una condición predeterminada ocurra. La ejecución automática contribuye a la reducción de controversias.

En el sector financiero, los smartcontracts pueden facilitar la emisión de valores a inversores directamente, acelerar el registro de datos entre las partes de una transacción, y actualizar los precios para reflejar el precio de mercado (Chamber of Digital Commerce, 2016).

A modo de analogía, los smartcontracts se parecen a una máquina expendedora de golosinas, donde el consumidor, una vez seleccionada la golosina deseada, previa introducción del dinero requerido por la máquina, pulsa el botón, iniciando una transacción irreversible, traducida en la recepción, por el consumidor, de la golosina selecta. 
Los contratos inteligentes son, particularmente, útiles para facilitar las transacciones en las que no existe confianza en las instituciones e intermediarios. Por ejemplo, son un sustituto útil cuando el cumplimiento del contrato depende de la actuación de un banco o de un funcionario del gobierno en un país extranjero, respecto del cual el beneficiario carece de confianza. En este escenario, permiten transacciones que, de otro modo, no se producirían.

\section{La blockchain: ¿enerva la posibilidad de que surjan disputas?}

La confianza que otorgan los smartcontracts, fruto de la tecnología blockchain, al reducir potencialmente la existencia de controversias, no se traduce en la imposibilidad de que estas ocurran, sobre todo si se consideran posibles errores humanos al crear o aplicar los contratos inteligentes.

Sin perjuicio de que en estos contratos impera una reducción de la voluntad humana, las personas, no obstante, son quienes escriben el. código con el que se redacta el smartcontract. Cualquier error en dicho código, afectará el desempeño del contrato. Los contratos inteligentes usualmente implican términos de codificación sumamente largos y complejos, en los que existe, sin dudas, un riesgo de error. Esos errores pueden dar lugar a resultados incorrectos.

En los mercados online, por ejemplo, es fundamental garantizar que las órdenes de transacción estén redactadas correctamente, puesto que los precios se actualizan constantemente y los usuarios envían solicitudes de compra inmediatas, afectándose el precio de compra en virtud de la relación oferta-demanda (Chu et al., 2016).

Otro ejemplo ocurrió con el "ataque DAO”. El DAO (Decentralized Autonomous Organization) constituye una vía para la creación de empresas bajo tecnología blockchain. Los usuarios compraban "fichas" (tokens) mediante criptodivisas y financiaban los proyectos que les interesaban, pudiendo retirar luego los beneficios resultantes de las inversiones. Un usuario, sin embargo, notó que esta función permitía un retiro de fondos continuo, previa actualización de los balances. Aprovechando ese error, pudo retirar 60 millones de dólares en criptomonedas. El creador del DAO explicó que, si en una línea del código informático, la redacción hubiera sido con una " $\mathrm{t}$ " minúscula, en lugar de una "T" mayúscula, se podría haber evitado el ataque. Ello muestra la potencialidad de errores que pueden ocurrir con estas tecnologías, los que darían lugar a múltiples controversias (Leising, 2017). 
Otro de los ejemplos que usualmente se utiliza para arrojar luz sobre la mecánica de los smartcontracts, es el de un contrato de seguro contra inundaciones. Allí, el pago a los asegurados se realiza automáticamente, cuando el nivel de precipitaciones alcanza un umbral predeterminado. En esos casos, las partes del contrato determinan un servicio meteorológico que suministrará los datos y un proveedor alternativo ante eventuales disfunciones del primero. Cuando el proveedor brinde datos que den cuenta de que se alcanzó el umbral predeterminado, configurando la inundación, el pago debería realizarse automáticamente. No obstante, la información del proveedor puede ser inexacta, generando un pago indebido o no generando un pago debido. Allí, nuevamente se hace necesario recurrir a métodos de solución de conflictos como el arbitraje, sobre la base, por ejemplo, de un enriquecimiento injusto.

Sin embargo, además de las circunstancias descritas, la entendible deficiencia de los operadores jurídicos en cuestiones tecnológicas de esta índole, lleva indefectiblemente a que estos, que negociaron o redactaron su contenido, no sean quienes escriben el código del smartcontract. Ello daría lugar a conflictos de interpretación, acerca de qué versión debe primar.

En relación con lo anterior, es menester precisar, no obstante, que no es lo mismo un smartcontract, que un smart legal contract. Al hablar de un contrato inteligente, nos referimos a un código de computadora que, ocurrida una o varias condiciones específicas, se ejecuta en un libro mayor distribuido (o cadena de bloques). Esto no necesariamente significa que se trate de un contrato legalmente exigible. Por el contrario, cuando se aplican estas tecnologías a un acuerdo jurídicamente exigible, de lenguaje tradicional, para su ejecución, estamos ante un smart legal contract, donde el código informático autoejecutable es capaz de verificar, facilitar, vigilar y hacer cumplir el acuerdo sin voluntad humana.

Otro problema radica en la dificultad de incorporar en códigos informáticos conceptos subjetivos como el de la buena fe o razonabilidad. Asimismo, no es unánimemente aceptado que se apliquen sanciones automáticamente, como esta tecnología permite. En primer lugar, según el foro aplicable, podría entenderse que la sanción aplicada de forma automática no es jurídicamente válida (Koulu, 2016). En segundo lugar, como se mencionó anteriormente, la dificultad de introducir en el código informático estándares subjetivos de valoración de hechos o pruebas, requeridos legalmente por los diversos foros, complica aún más el panorama. En adición a ello, 
diversos países contemplan la situación de los consumidores, parte débil del contrato, determinando la no vinculatoriedad de las cláusulas consideradas injustas (English Consumer Rights Act, 2015). Por tanto, cláusulas que sancionen automáticamente ante hipótesis de incumplimiento del consumidor, en smartcontracts de consumo (por ejemplo) podrían considerarse inválidas, por crear un desequilibrio entre las partes.

Esto constituye un desafío para los operadores jurídicos en la utilización de estas tecnologías, debiendo adaptarlas a las peculiaridades de cada negocio, sobremanera cuando la legislación aplicable otorgue un trato especial a una de las partes.

Debido a las circunstancias descritas y por muy buenos augurios que existan sobre estas tecnologías, la posibilidad de disputas, como en toda acción que involucre el comportamiento humano, está presente.

\section{¿Son contratos?: problemas de calificación}

Pese a su denominación, no es unánimemente aceptada la naturaleza contractual de los smartcontracts. Naturalmente, no es el nombre, sino su adecuación a una figura jurídica, lo que determina ante qué tipo de negocio estamos. Que los contratos inteligentes sean efectivamente contratos, dependerá de la legislación de cada jurisdicción.

Algunos niegan la naturaleza contractual de los contratos inteligentes (Lim, 2016), alegando que son meros programas informáticos. En efecto, no existen relaciones legales en el espacio o vacío. Sin embargo, dicha afirmación debe matizarse con las precisiones ya efectuadas. Del cotejo entre el contrato inteligente en concreto y los elementos constitutivos del contrato requeridos por una legislación determinada, se sabrá si se está ante una figura jurídica contractual u otra, si no hay adecuación.

Algunos smartcontracts están escritos únicamente en códigos informáticos, mientras que otros están acompañados por un contrato tradicional, redactado en lenguaje natural. En la segunda hipótesis, la posibilidad de negarle naturaleza contractual parece desaparecer.

The Law Society, organismo profesional independiente de abogados de Inglaterra y Gales, a través del panel LawTech, emitió una declaración en la que consideró que los smartcontracts son, en principio, capaces de satisfacer los requisitos constitutivos de un contrato bajo la ley inglesa, pero el efectivo cumplimiento de este dependerá, en definitiva, de circunstancias fácticas como la conducta de las partes o el lenguaje utilizado. El grupo de expertos discrepó, por su parte, con la exigencia de un contrato 
adicional, en lenguaje natural, que sirva de envoltorio legal para que el contrato inteligente tenga fuerza vinculante, dado que el derecho inglés no exige para los contratos una forma particular. Así, concluyen que los contratos inteligentes que existen puramente en el código informático siguen siendo capaces de dar lugar a obligaciones legales vinculantes, en tanto se cumplan los requisitos constitutivos de los contratos bajo la ley aplicable (LawTech, 2019).

Por su parte, potenciales conflictos derivados de la ingeniería de estos acuerdos pueden suscitarse. En efecto, quizás resulte difícil establecer quiénes son los actores de la red, dónde se hallan y cuáles han sido exactamente sus acciones, lo que tiene innegables implicancias jurídicas, tanto a nivel local como eventualmente desde la perspectiva del derecho internacional, a efectos de determinar responsabilidades, o el foro y ley aplicable en una disputa (debe precisarse que estos conflictos no ocurren en toda cadena de bloqueo, pues en algunas los nodos están situados en una única jurisdicción determinada).

Como señalaron expertos de la Unión Europea, ninguno de estos conflictos es, no obstante, insuperable (Foro y Observatorio de Blockchain de la Unión Europea, 2019). En primer lugar, en virtud de la evolución de las herramientas legales y regulatorias al auxilio de las autoridades (por ejemplo, a través del internet, las autoridades recurren a ciertos puntos de acceso que les permite supervisar y hacer cumplir la normativa aplicable, hasta en entornos sumamente descentralizados y sin permisos). La propia tecnología blockchain ha desarrollado herramientas al auxilio de las autoridades, con métodos que permiten el "traspaso del velo" para la identificación de los participantes en la red.

En segundo lugar, adaptando el marco regulatorio local e internacional a estas nuevas tecnologías para trabajar en soluciones que mitiguen estos problemas, haciendo parte de dicho proceso, a toda la comunidad.

\section{Arbitraje: la fórmula ideal}

Como se explicó, la aparición de controversias no es ajena a estos contratos. A modo de ejemplo, supóngase un sujeto $X$, propietario de un terreno que quiere enajenar a un sujeto Y, mediante una solución basada en tecnología blockchain. A través de esta, X ejecuta un smartcontract que transfiere la ficha electrónica que representa su porción de tierra al sujeto Y. Cabe la aclaración, esas fichas electrónicas (tokens), son unidades de valor que una organización crea para gobernar su modelo de negocio (Mougayar, 2016). 
Continuando con el ejemplo, la ficha se transfiere de $\mathrm{X}$ a $\mathrm{Y}$, estando ahora $\mathrm{Y}$, en la blockchain, representado como el nuevo propietario del terreno. No obstante, las legislaciones suelen exigir requisitos de solemnidad para transferencias de esa naturaleza, circunstancia que quienes diseñaron el sistema de smartcontracts no previeron. Como resultado, Y no sería reconocido por la justicia como propietario. Si bien los Tribunales no podrían modificar el resultado de la transferencia en la blockchain (dado que, como se mencionó, los datos almacenados en el registro son inmutables), podrían, no obstante, surgir disputas relativas a la propiedad del terreno, y allí la postura del juzgador estará determinada por su lectura sobre las normas aplicables a los hechos, y no por lo que surja de visualizar la blockchain (Clifford Chance, 2017).

En esos casos es fundamental que los abogados de las partes, en conjunto con los asesores informáticos que diseñan el contrato inteligente, prevean las peculiaridades del negocio a efectos de prever situaciones indeseables como la mencionada, que eventualmente podrían acarrear la nulidad del negocio.

El arbitraje es, en primer lugar, útil para resolver estas disputas, dadas sus similitudes con los smartcontracts. En efecto, ambos operan de forma descentralizada: el arbitraje es deslocalizado, lo que importa separación, en muchos aspectos, de las limitaciones de las leyes locales. Los laudos, se ha sostenido, no integran ningún ordenamiento local, siendo, por el contrario, decisiones de justicia trasnacional (French Court of Cassation, 2017). De igual forma, los smartcontracts se incorporan a un libro contable distribuido y determinar la ubicación de los elementos contractuales, como el lugar de cumplimiento a efectos de determinar el foro competente, puede ser tan difícil como imposible.

Por su parte, controversias que involucran aspectos sumamente técnicos, requieren de procedimientos con reglas flexibles, contrariamente a la tradición judicial de los Estados. El arbitraje es por naturaleza flexible, pudiendo las partes nombrar árbitros especializados en la materia y adoptar el procedimiento a las particularidades de estas tecnologías.

Finalmente, cabe resaltar que en estos contratos, el secreto suele ser un valor esencial. El arbitraje es el medio de resolución de conflictos, por excelencia, adecuado a dicho fin (Born, 2014). 


\section{Arbitraje "off-chain"}

Una de las formas de resolver las controversias es a través del arbitraje tradicional, institucional o ad hoc. En efecto, algunas plataformas sugieren la inclusión de cláusulas de arbitraje para posibles disputas (Ethereum).

En ese sentido, la redacción de un reglamento o ley modelo de arbitraje, especialmente adaptada a disputas de esta naturaleza, sería particularmente útil en caso de que las partes pacten someter sus eventuales controversias a dichos instrumentos aggiornados a estas tecnologías.

De igual forma, en la selección de árbitros podrían elegirse a aquellos más idóneos en el rubro tecnológico, que comprendan adecuadamente la estructura del negocio. Colabora a dicho fin la capacitación de los operadores jurídicos (incluyendo a los árbitros, abogados, secretarios de tribunal, etc.) y una mayor digitalización del proceso.

Finalmente, cabe resaltar que el laudo arbitral podría registrarse en la cadena, siendo la decisión autoejecutable. El problema en este caso, radica en la forma de lograr esa incorporación. A tales efectos, sería conveniente que las partes prevean el arbitraje en el contrato, así como la posterior recepción del laudo en el contrato inteligente.

Luego, y en la medida en que la información almacenada en la blockchain es inmanipulable y auténtica, una de las partes podría brindar acceso a la blockchain al tribunal. El tribunal debería cotejar que el acuerdo arbitral en forma de comunicaciones digitales cumple con los requisitos legales de escritura establecidos por la Convención de Nueva York.

La dificultad radica, sin embargo, en que de acuerdo con el art. V de la Convención de Nueva York, luego de que una parte presenta el fallo ante un tribunal solicitando la ejecución, la contraparte puede oponerse con base en las causales de dicha disposición, dando lugar a una nueva etapa del procedimiento, en donde las partes argumentarán a favor o en contra de la ejecución. Por tanto, si bien cargar el acuerdo arbitral y el laudo a la blockchain puede agilizar su ejecución y abaratar costos, esto no necesariamente será así.

\section{Arbitraje "on-chain"}

El arbitraje en cadena implica que la blockchain utilizada para la transacción, cuenta con una suerte de autoridad central para arbitrar las disputas. La ventaja de este procedimiento es que el arbitraje se integra a la cadena, siendo posible, por ejemplo, 
pausar la transacción si es necesario y administrar eventuales recursos. Asimismo, un algoritmo podría resolver la controversia con base en el examen de disputas similares. Estas hipótesis, precisamente, están previstas en la plataforma Kleros.

De forma alternativa, el arbitraje en cadena podría realizarse mediante la unión de individuos al blockchain, que se incluyan en un grupo de posibles árbitros, los cuales podrían ser seleccionados por las partes o por el propio algoritmo en función de un mecanismo preestablecido. Constituido el grupo de expertos, recibirán las pruebas y datos sobre la controversia y contarán con un plazo para fallar. El fallo se incorporará a la cadena y se ejecutará de forma automática bajo el smartcontract.

\section{Problemas con el reconocimiento y la ejecución del laudo}

Uno de los principales problemas de la blockchain y su relación con el arbitraje es aquel que puede surgir al momento de buscarse el reconocimiento o la ejecución del laudo. La búsqueda de la eficiencia y celeridad debe ser una máxima en cualquier sistema de resolución de disputas. No obstante, su consecución no puede obtenerse a cambio de postergar uno de los valores máximos, cual es la justicia.

Así, en primer lugar, cabe considerar la hipótesis del arbitraje off-chain, vale decir, el arbitraje tradicional. En ese caso, se abren dos posibilidades: que se arribe a un laudo cuya ejecución se busca por la vía estatal o que dicho laudo se incorpore al blockchain y se ejecute automáticamente.

Si el laudo busca ejecutarse por la vía estatal, no habría inconvenientes más que aquellos que también se presentan tratándose de procedimientos en los que estas tecnologías no están involucradas.

Más compleja es la hipótesis en la que la ejecución del laudo no se busca por las vías tradicionales, sino, por el contrario, de forma automática, incorporándolo a la cadena de bloques. Cabe plantearse, en ese caso, cómo podría la contraparte abogar por la no ejecución de un laudo que, precisamente, se pretende ejecutar automáticamente. A criterio del autor, siempre debería estar abierta la vía para que, a través de la justicia estatal, el ejecutado pueda pedir una revisión del laudo y, en ese caso, dado que la transferencia de fondos ya fue efectuada, podría condenarse a la contraparte a que los devuelva. En cualquier caso, deberá estarse a la interpretación de las distintas cláusulas del contrato.

En segundo lugar, debe considerarse la hipótesis de laudos a los que se arribó a través de un arbitraje on-chain. El arbitraje en cadena puede desarrollarse de dos maneras: 
de forma automática, en base a una tecnología predictiva y utilizando patrones, o mediante un sistema de jurado o árbitros.

En los casos de arbitrajes automáticos, el laudo se ejecutará automáticamente. No caben dudas de que, al menos en el contexto actual, sería siempre posible acudir a la justicia ordinaria a efectos de revertir el statu quo, ya que difícilmente las legislaciones permitirían que la suerte de las personas se determine en ausencia de voluntad humana. Este tipo de resolución de conflictos puede ser útil para cuestiones de índole administrativa. No obstante, la apreciación de los hechos bajo estándares subjetivos, así como la valoración de la prueba y la aplicación de principios que orientan al juzgador al momento de fallar, salvo excepciones, son factores que no es posible sortear.

Constituye un enorme desafío, entonces, lograr incorporar patrones subjetivos en la resolución de conflictos digital, proceso en el que la inteligencia artificial puede contribuir sobremanera.

Un ejemplo de ejecución automática de laudos dictados on-chain lo constituye el procedimiento utilizado por las redes sociales para eliminar el contenido establecido como perjudicial, como archivos íntimos difundidos sin consentimiento, violaciones a derechos de autor y fake news. La sanción puede ir desde la eliminación del contenido hasta la eliminación de la cuenta.

En el caso de un arbitraje on-chain, con un sistema de jurado o tribunal integrado por humanos, la ejecución automática del laudo es, nuevamente, revisable en la vía estatal. Si, por el contrario, el laudo busca ejecutarse ante un tribunal estatal, los problemas aparecen en torno a su ejecutabilidad, por el posible incumplimiento de los requisitos establecidos por la Convención de Nueva York.

Ambos tipos de arbitraje, tanto off-chain como on-chain, pueden presentar dificultades particulares en relación al cumplimiento de los estándares exigidos por la Convención de Nueva York.

\section{Escrituración de la cláusula arbitral}

La Convención de Nueva York, instrumento de recepción universal que orienta a los magistrados al enfrentarse a laudos cuya ejecución se solicita, exige que la cláusula o acuerdo arbitral conste "por escrito". Luego, dispone en su artículo II.2, que ello significa "una cláusula compromisoria incluida en un contrato o un compromiso, firmados por las partes o contenidos en un canje de cartas o telegramas". Las circunstancias descritas, no 
obstante, no son exhaustivas. En efecto, doctrinaria y jurisprudencialmente se entiende por cumplido el requisito de escritura de una cláusula arbitral, a través de medios tecnológicos. Lo que es relevante es que del conjunto de elementos de que dispone el tribunal surja el consentimiento de las partes para someter sus eventuales disputas a arbitraje (England y Wales Court of Appeal - Civil Division, 2012).

La Recomendación relativa a la interpretación del párrafo 2) del artículo II y del párrafo 1) del artículo VII de la Convención de Nueva York, establece que el requisito de escritura del acuerdo arbitral incluye a los medios electrónicos de comunicación. Por su parte, la Ley Modelo Uncitral de 1985 (con las enmiendas de 2006), establece en el Capítulo II, Opción I, art. 7(4): “El requisito de que un acuerdo de arbitraje conste por escrito se cumplirá con una comunicación electrónica si la información en ella consignada es accesible para su ulterior consulta". Idéntica solución establece la Convención de las Naciones Unidas sobre la Utilización de las Comunicaciones Electrónicas en los Contratos Internacionales en su art. 9(2): “Cuando la ley requiera que una comunicación o un contrato conste por escrito, o prevea consecuencias en el caso de que eso no se cumpla, una comunicación electrónica cumplirá ese requisito si la información consignada en su texto es accesible para su ulterior consulta" (Convención sobre Comunicaciones Electrónicas, 2007).

Por tanto, tratándose de contratos inteligentes en los que podemos acceder al código fuente, evidenciada la intención de las partes de arbitrar sus disputas, se cumplirá el requisito de escritura.

\section{Capacidad de las partes}

La falta de capacidad es, bajo la Convención de Nueva York, motivo fundante de la denegación de reconocimiento o ejecución del laudo. Constituye un desafío, entonces, resolver los casos en los que no sea posible arribar a la identidad de las partes, o a la ley aplicable a efectos de observar si estas eran capaces de contratar.

\section{Violación de normas de orden público}

También podría denegarse el reconocimiento o ejecución del laudo en caso de que el tribunal estatal estime que se han violado las disposiciones de orden público de su país. Esto ocurriría, por ejemplo, si el laudo condena al pago de daños y perjuicios a una parte por el incumplimiento de un acuerdo anticompetitivo y, en dicho Estado, las disposiciones 
sobre derecho de la competencia tienen carácter de orden público. El incumplimiento de principios elevados a esa categoría, como el favor debitoris, también ameritarían la no ejecución del laudo.

\section{Necesidad de acompañar la sentencia y el acuerdo arbitral}

Finalmente, la Convención de Nueva York establece en su artículo IV, a efectos de solicitar el reconocimiento y ejecución del laudo, que es menester acompañar la demanda con "el original debidamente autenticado de la sentencia o una copia de ese original" y "el original del acuerdo a que se refiere el artículo II, o una copia". Sería imperioso, entonces, que los árbitros que dictaron el laudo on-chain, dicten el fallo en un formato que habilite a su ejecución por la vía estatal. En la segunda hipótesis, nuevamente, se vuelve necesario acompañar el contrato con una prueba fehaciente de este (o del acuerdo arbitral, si está separado del contrato), como, por ejemplo, a través de un documento electrónico, definido como la "representación digital de actos o hechos, con independencia del soporte utilizado para su fijación, almacenamiento o archivo" (Ley $\mathrm{N}^{\circ}$ 18.600, art. 2).

\section{Initial Coin Offering: criptomonedas y arbitraje}

Otra de las posibles aplicaciones del arbitraje en materia de tecnología blockchain, surge con las criptomonedas.

Las criptomonedas son activos digitales descentralizados cuyo valor no está respaldado por una institución tercera, como un banco central. Son activos representados en la blockchain. En lugar de depender de aquel tercero para registrar las transacciones, la cadena de bloques aprovecha los grandes recursos de las redes peer-to-peer para verificar y confirmar cada transacción en criptomoneda.

Uno de los usos dados a las criptodivisas es como vía para aglutinar el capital de inversión, mediante una oferta inicial de monedas ("ICO": Initial Coin Offering). Las ICO son un instrumento de financiación colectiva que suelen basarse en la misma estructura: una empresa busca capital para desarrollar una idea, basándose en la tecnología blockchain, pero en lugar de recurrir a los canales tradicionales, la empresa anuncia su proyecto a través de la red, en el que se describe el programa de crecimiento tecnológico y comercial futuro y se fija una cantidad mínima de fondos necesarios. Se invita a los inversores a financiar el proyecto mediante la compra de "fichas/tokens". El 
procedimiento por el que los inversores pagan por las fichas y las reciben está normalmente regulado por las condiciones generales de la ICO. Una vez concluida la ICO, el capital debe ser utilizado por la empresa para iniciar el desarrollo siempre que se cumpla el umbral mínimo; en cambio, si los fondos recaudados no alcanzan el umbral, los inversores deben recibir inmediatamente su capital de vuelta.

Las fichas pueden funcionar como una acción, otorgando al comprador participaciones en el capital de la empresa emisora, con derecho a voto y dividendos. Asimismo, pueden operar como puntos ganados en un programa de recompensas, permitiendo al dueño acceder a determinados beneficios ofrecidos por la empresa, como bienes o servicios.

Tal como ocurre con las acciones, puede que el valor de la criptomoneda aumente si la empresa tiene éxito. Así, puede negociarse a nivel global en bolsas que manejen criptomonedas. Y como en cualquier empresa en la que los inversores ponen en peligro los fondos, las incertidumbres en cuanto a la asignación del riesgo, o la base sobre la que se asumió, pueden dar lugar a controversias. Por ejemplo, para atraer la inversión, una empresa que lanza una ICO brindará información sobre su negocio, que puede incluir un memorando o prospecto de oferta, análogo a una circular de oferta emitida por una empresa dedicada a la emisión de bonos. Las cláusulas arbitrales podrían ser el mecanismo razonable de solución de controversias, para incluir en esos documentos, dado los eventuales incumplimientos a las cláusulas de esos memorandos, así como posibles reclamaciones en función de la manera en que se llevó a cabo la ICO. Sería el caso, por ejemplo, de una empresa emisora, que, dada la congestión de la red, debido a una gran demanda, decide mantener abierta la oferta durante más tiempo que el inicialmente previsto.

En ese caso, los primeros inversores podrían reclamar, en virtud de que se permitió que los fondos recaudados sucedieran al objetivo máximo del emisor, con la consiguiente disminución del valor de las criptomonedas compradas antes de alcanzar ese objetivo. Asimismo, pueden suscitarse litigios vinculados a cuestiones de publicidad falsa, competencia desleal, fraude de valores, incumplimiento de contrato y de leyes de consumo (Chan y Maynard, 2017). En función del perfil internacional de los inversores de la ICO, el arbitraje se postula, nuevamente, como el medio ideal para la resolución de los conflictos. 


\section{Arbitraje de inversiones}

En adición a las reflexiones anteriores, cabe señalar que el arbitraje de inversiones tampoco está exento de verse influido por el uso de las nuevas tecnologías. Países como Bielorrusia han sancionado leyes permisivas, autorizando las ICO y estableciendo que el uso e intercambio de las criptomonedas y los smartcontracts, son actividades comerciales legítimas. Así, el país en cuestión tomó una medida que contribuye a efectos de atraer inversores extranjeros que utilicen estas tecnologías. Venezuela, por su parte, se atrevió a emitir su propia criptomoneda. Es dable cuestionarse, entonces, si estos activos digitales pueden constituir inversiones protegidas, a la luz del derecho internacional.

La elaboración de criptomonedas como bitcoin es costosa e involucra un proceso prolongado en el tiempo. La actividad de "cripto-minería" podría, por su naturaleza, asimilarse a una planta industrial, en virtud de las definiciones tradicionales de los tratados bilaterales de inversión (BIT: Bilateral Investment Treaty). Por tanto, pueden constituir inversiones protegidas y cualquier cambio legislativo constituye un potencial motivo fundante de reclamaciones.

No es tan claro el panorama con relación a si la propia moneda digital puede catalogarse como inversión protegida. Es necesario precisar que, con excepción de algunas legislaciones, estos activos, no obstante denominarse criptomonedas, no constituyen monedas de curso legal. Ahora bien, así como se ha analizado la posibilidad de que el dinero mantenido en una cuenta bancaria constituya inversión protegida, podría serlo, también, un activo digital "guardado" en una cartera (claves privadas necesarias para acceder a la propia criptodivisa).

Naturalmente que, para que se trate de una inversión protegida, el activo criptográfico debería estar en el territorio o bajo el dominio del Estado receptor, lo que complica el panorama tratándose de tecnología blockchain, deslocalizada por definición. De todas formas, es posible alegar que determinados derechos y obligaciones vinculados al activo sólo surgen en ese territorio, $u$ otras conexiones que acrediten que el activo está ubicado en este (Kerjean y Terrien, 2018). 


\section{Conclusiones}

La tecnología de las cadenas en bloque está revolucionando el mundo de los negocios y, también, la forma de pensar. A través de su uso, se pueden mitigar muchas ineficiencias. Su utilidad práctica deriva en disminución de tiempos y costos. Como se desarrolló, estas consecuencias están repercutiendo en el ámbito legal. Por un lado, inciden en la forma en que se prestan los servicios por los operadores jurídicos. De igual forma, pueden tener beneficios muy positivos en la resolución de disputas.

El futuro, sin embargo, es incierto. Lo que es indudable, es que las utilidades de la blockchain no obstan a que su aplicación deba efectuarse con suma cautela en el ámbito del arbitraje.

Existe una gran brecha entre el funcionamiento de la tecnología, y las distintas legislaciones. Sin perjuicio de que algunos instrumentos normativos pecan de conservadores, la realidad es que, en general, las trabas impuestas se justifican en aras de preservar las garantías inherentes a las personas por su calidad de tales, como lo son aquellas integrantes del debido proceso, de raigambre constitucional.

De momento, es menester esperar y observar cómo evoluciona esta tecnología en el ámbito de la contratación y de la resolución de disputas. El arbitraje es el método ideal para resolver estos problemas, por tratarse de un sistema deslocalizado, como la propia cadena de blockchain. Es, por excelencia, el procedimiento más adaptable al cambio. En ese proceso de adaptabilidad, debe buscarse la forma de lograr laudos ejecutables, anclando los contratos inteligentes a un marco jurídico válido y adecuado. La autoejecutabilidad de estos instrumentos sólo dificulta el panorama, aumentando el riesgo jurídico. Mantener los principios tradicionales de la formación y cumplimiento de contratos resulta imperioso.

Sin perjuicio de los desafíos que estas tecnologías plantean, la realidad evidencia que se observa, a nivel mundial, un enorme potencial para el ámbito transaccional. La comunidad arbitral, por tanto, no debe descuidarse. Tiene que seguir de cerca estos avances para adaptarse y proporcionar el mejor marco posible para resolver cualquier conflicto. 


\section{Referencias}

Ast, F. (2017). Kleros, a Protocol for a Decentralized Justice System. Recuperado el 24 de marzo de 2020 de https://medium.com/kleros/kleros-a-decentralized-justice-protocolfor-the-internet-38d596a6300d

Ast, F., George, W., y Lesaege, C. (2019). Kleros Short Paper v1.0.7. Recuperado el 5 de octubre de 2020 de https://kleros.io/whitepaper.pdf

Bacon, J., Michels, J., Millard, C., y Singh, J. (2017). Blockchain Demystified. Queen Mary School of Law Legal Studies Research, Paper No. 268/2017. Recuperado el 07 de abril de 2020 de https://ssrn.com/abstract=3091218.

Bartoletti, M. y Pompianu, L. (2017). An empirical analysis of smart contracts: platforms, applications, and design patterns. Financial Cryptography Workshops. Recuperado el 03 de abril de 2020 de https://arxiv.org/pdf/1703.06322.pdf

Born, G. (2014). International Commercial Arbitration. Kluwer Law International.

Chan, E. y Maynard, S. (2017). Decrypting Cryptocurrencies: Why Borderless Currencies May Benefit from Borderless Dispute Resolution. Kluwer Arbitration Blog. Recuperado el 04 de abril de 2020 de http://arbitrationblog.kluwerarbitration.com/2017/11/02/decrypting-cryptocurrenciesborderless-currencies-may-benefit-borderless-dispute-resolution/

Chu, D.-H., Luu, L., Hobor, A., Olickel, H. y Saxena, P. (2016, octubre). Making Smart Contracts Smarter. Presentado en Proceedings of the 2016 ACM SIGSAC Conference on Computer and Communications Security, Viena, Austria.

Clifford Chance. (2017). Are smart contracts contracts? Talking Tech looks at the concepts and realities of smart contracts. Recuperado el 04 de abril de 2020 de https://talkingtech.cliffordchance.com/en/emerging-technologies/smart-contracts/aresmart-contracts-contracts.html

Demartini, C., Gatteschi, V., Lamberti, F., Pranteda, C. y Santamaría, V. (2018). Blockchain and Smart Contracts for Insurance: Is the Technology Mature Enough?. Future Internet.

De Filippi, P. y Wright, A. (2015). Decentralized Blockchain Technology and the Rise of Lex Cryptographia. Recuperado el 08 de abril de 2020 de https://papers.ssrn.com/sol3/papers.cfm?abstract_id=2580664

Fenwick, M., Kaal, W. y Vermeulen, E. (2017). Legal Education in the Blockchain Revolution. Recuperado el 05 de abril de 2020 de http://www.jetlaw.org/wpcontent/uploads/2017/12/1_Kaal-Article_Final-Review-Complete_Approved.pdf

Foro y Observatorio de Blockchain de la Unión Europea (2019). Legal and Regulatory Framework of Blockchains and Smart Contracts. Recuperado el 13 de septiembre de 2020 de https://www.eublockchainforum.eu/sites/default/files/reports/report_legal_v1.0.pdf

Gupta, M. (2017). Blockchain For Dummies. IBM. 
Hodge, M. y Neally, D. (2018). Blockchain in the Courts. First Annual Dennis Karjala Memorial Workshop. Recuperado el 05 de abril de 2020 de http://blogs.asucollegeoflaw.com/lsi/files/2018/12/First-Annual-Karjala-WorkshopReport.pdf

Katsh, E. y Rabinovich-Einy, O. (2017). Digital justice: technology and the internet of disputes. Oxford Scholarship Online.

Kerjean, A. y Terrien, A. (2018). Blockchain and Cryptocurrencies: The New Frontier of Investment Arbitration? Kluwer Arbitration Blog. Recuperado el 08 de abril de 2020 de http://arbitrationblog.kluwerarbitration.com/2018/10/18/blockchain-andcryptocurrencies-the-new-frontier-of-investmentarbitration/?doing_wp_cron=1586526570.1271018981933593750000

Koulu, R. (2016). Blockchains and Online Dispute Resolution: Smart Contracts as an Alternative to Enforcement. Scripted Review, 13(1). Recuperado el 5 de abril de 2020 de https://script-ed.org/article/blockchains-and-online-dispute-resolution-smartcontracts-as-an-alternative-to-enforcement/

LawTech UK - Delivery Panel. (2019). Cryptoassets, DLT and smart contracts - UK Jurisdiction Taskforce consultation. Recuperado el 10 de abril de 2020 de https://www.lawsociety.org.uk/campaigns/lawtech/news/cryptoassets-dlt-and-smartcontracts-ukjt-consultation/

Leising, M. (2017). The Ether Thief. Bloomberg. Recuperado el 10 de abril de 2020 de https://www.bloomberg.com/features/2017-the-ether-thief/

Lim, C. (2016). Smart Contracts: Bridging the Gap Between Expectation and Reality. University of Oxford.

Mougayar, W. (2016). The business Blockchain promise, practice, and application of the next Internet technology. John Wiley \& Sons Limited.

Pastorino, C. (2018). Blockchain: qué es, cómo funciona y cómo se está usando en el mercado. Recuperado el 20 de marzo de 2020 de https://www.welivesecurity.com/laes/2018/09/04/blockchain-que-es-como-funciona-y-como-se-esta-usando-en-elmercado/

Pilkington, M. (2016). Blockchain Technology: Principles and Applications. Research Handbook on Digital Transformations, editado por Xavier Olleros, F. y Majlinda Zhegu, E. https://ssrn.com/abstract=2662660

Smart Contracts Alliance - In collaboration with Deloitte. (2016). Smart Contracts: 12 Use Cases for Business \& Beyond A Technology, Legal \& Regulatory Introduction. Chamber of Digital Commerce. Recuperado el 28 de marzo de 2020 de https://digitalchamber.org/wp-content/uploads/2018/02/Smart-Contracts-12-Use-Casesfor-Business-and-Beyond_Chamber-of-Digital-Commerce.pdf

Weber, R. (2018). An Advisor's Introduction to Blockchain. Journal of Financial Service Professionals, 72(6). 


\section{Fallos citados}

Société PT Putrabali Adyamulia v. Société Rena Holding et Société Moguntia Est Epices. 29 de junio de 2007. Cour de Cassation de Francia. Caso "05-18.053".

Sulamérica Cia Nacional de Seguros S.A. v. Enesa Engenharia S.A. 16 de mayo de 2012. Court of Appeal (Civil Division) de Inglaterra y Gales. Caso “A3/2012/0249”.

\section{Normativa citada}

Consumer Rights Act de Inglaterra. 2015. Disponible en: http://www.legislation.gov.uk/ukpga/2015/15/contents/enacted.

Convención de las Naciones Unidas sobre la Utilización de las Comunicaciones Electrónicas en los Contratos Internacionales. 2007. Disponible en: https://www.uncitral.org/pdf/spanish/texts/electcom/06-57455_Ebook.pdf.

Convención sobre el Reconocimiento y la Ejecución de las Sentencias Arbitrales Extranjeras. 1958. Disponible en: https://www.uncitral.org/pdf/spanish/texts/arbitration/NYconv/New-York-Convention-S.pdf.

Ley $\mathrm{N}^{\circ}$ 18.600. Disponible en: https://www.impo.com.uy/bases/leyes/18600-2009/.

Ley Modelo de la Comisión de las Naciones Unidas para el Derecho Mercantil Internacional, sobre Arbitraje Comercial Internacional. 1985, con las enmiendas de 2006. Disponible en: https://uncitral.un.org/sites/uncitral.un.org/files/media-documents/uncitral/es/0787001_ebook.pdf.

Recomendación relativa a la interpretación del párrafo 2) del artículo II y del párrafo 1) del artículo VII de la Convención sobre el Reconocimiento y la Ejecución de las Sentencias Arbitrales Extranjeras de 1958. 2006. Disponible en:

https://uncitral.un.org/sites/uncitral.un.org/files/media-documents/uncitral/es/a2s.pdf.

Reglamento de Arbitraje de la Asociación Americana de Arbitraje (AAA) - Centro Internacional para la Resolución de Disputas (ICDR). 2014. Disponible en: https://www.icdr.org/sites/default/files/document_repository/ICDR_Rules.pdf.

Reglamento de Arbitraje de la Cámara Internacional de Comercio. 2017. Disponible en: https://iccwbo.org/publication/arbitration-rules-mediation-rules-spanish-version/.

\section{Contribución autoral}

a) Concepción y diseño del trabajo; b) Adquisición de datos; c) Análisis e interpretación de datos; d) Redacción del manuscrito; e) revisión crítica del manuscrito.

I. T. ha contribuido en a, b, c, d, e.

\section{Editor científico responsable}

Dra. María Paula Garat 